\title{
Affect in science communication: A data-driven analysis of TED talks on YouTube
}

\author{
Olivia Fischer*1, Loris T. Jeitziner*1, and Dirk U. Wulff ${ }^{1,2}$ \\ *Authors contributed equally; order determined by coin flip. \\ ${ }^{1}$ University of Basel \\ ${ }^{2}$ Max Planck Institute for Human Development
}

\begin{abstract}
Science communication is evolving as it is increasingly directed at the public rather than academic peers. Understanding the circumstances under which the public engages with scientific content is therefore crucial to improving science communication. In this article, we investigate the role of affect on audience engagement with a modern form of science communication: TED talks on the social media platform YouTube. We examined how affect valence-a net positive or negative affect — and density — the proportion of affective words—are associated with a talk's popularity-reflecting views and likes-and polarity-reflecting dislikes and comments. We found that the valence of TED talks was associated with both popularity and polarity, with positive valence being linked to higher talk popularity and lower talk polarity. Density, on the other hand, was only associated with popularity, with higher affective density being linked to higher popularity - even more so than valence-but not polarity. Moreover, we observed that the association between affect and engagement was moderated by talk topic, but not whether the talk included scientific content or not. We discuss possible mechanisms and implications of our findings for increasing the effectiveness of science communication.
\end{abstract}

Keywords: science communication, TED talks, social media, YouTube, sentiment analysis

The digital age presents great opportunities for and challenges to science communication. Communication hubs such as Twitter, Facebook and YouTube deliver unprecedented reach for scientific content and interaction with the public (Collins et al., 2016), thereby making science more accessible for scientists and laypeople alike. With engagement tools such as likes, dislikes, comments, or shares, the public now no longer simply consume scientific content but take on a participatory role in disseminating it. Conversely, if not deemed engaging enough by the public, scientific content may never reach a large audience. In the over-saturated and highly competitive environment of social media, how can scientists make their voices heard?

Science communication via social media differs in at

Olivia Fischer (D https://orcid.org/0000-0002-7815-9797 Loris Jeitziner (D) https://orcid.org/0000-0001-7941-5003 Dirk U. Wulff (D) https://orcid.org/0000-0002-4008-8022

We are grateful to Laura Wiles for editing the manuscript. This work was supported by a grant from the Swiss Science Foundation (100015_197315) to Dirk U. Wulff.

Correspondence concerning this article should be addressed to Dirk U. Wulff, Department of Psychology, University of Basel, Missionsstrasse 60-62, 4055 Basel, Switzerland. E-mail: dirk.wulff@gmail.com least two important respects from traditional peer-to-peer science communication. First, the social media audience tends to consume content more superficially (Boczkowski et al., 2017), implying that surface-level aspects such as the choice of language likely become more important in gaining a competitive advantage. Second, social media facilitates both direct and indirect sharing of content, with the latter being mediated by recommender systems (Covington et al., 2016). These differences introduce strong positive feedback between user engagements, which can greatly amplify the reach for especially engaging content (Aldous et al., 2019, Davidson et al., 2010, Hoiles et al., 2017). This means that scientists rely on non-scientists to propagate their messages on social media, which incentivizes scientists to pay attention to aspects of science communication that make it more engaging.

In this article, we investigate affect as one aspect of science communication that may be instrumental for effective science communication (Milkman \& Berger, 2014). Past work has found that New York Times articles using more affect-rich language were more likely to make the New York Times' most-emailed list (e.g., Berger \& Milkman, 2012). There is also evidence that scientific findings that are described in a more affective manner are more likely to be shared (Milkman \& Berger, 2014) and tend to garner more citations (Fronzetti Colladon et al., 2020). However, the po- 
tential link between affect and engagement as a driver of dissemination has not been systematically investigated for social media-based science communication (see Davies, 2019, Davies et al., 2019; Osseweijer, 2006). We aim to fill this gap with a data-driven analysis of engagement with TED talks on the social media platform YouTube.

TED talks are video recordings of short presentations on technology, entertainment, and design, including many talks on basic and applied science. Given these characteristics, TED talks are regarded and studied as a modern form of science communication (e.g., Gheorghiu et al., 2020, MacKrill et al., 2021; Sugimoto \& Thelwall, 2013; Verjovsky \& Jurberg, 2020). On their webpage (www.ted.com), the TED organization provides curated transcripts for all talks, which can be used to derive their affective features. In addition to the organization's own web-based platform, TED talks are also shared via the organization's channel on YouTube. There, TED talks have gathered a total of 19.8 million subscribers and over two billion video views 1 The popularity of TED talks on YouTube, which reflects that they are targeted at a lay audience and recruit lower levels of jargon (Rakedzon et al., 2017; Sharon \& Baram-Tsabari, 2014), has produced a rich data trove on public engagement that can be linked to the talks' affective features.

By investigating the role of affect in driving engagement with TED talks on YouTube, we contribute to a growing body of work on social media-based science communication (see Allgaier, 2020, Brossard, 2013, Kohler \& Dietrich, 2021) and, in particular, science communication on YouTube. Past work has focused on understanding the role of characteristics of the video presenters for user engagement, including their gender (Amarasekara \& Grant, 2019), professional background, and perceived authenticity (Kaul et al., 2020), as well as on understanding the psychological processes on the side of the recipient, for instance, by means of tracking eye-movements (Boy et al., 2020) or analyzing the semantic and emotional content of YouTube comments (Amarasekara \& Grant, 2019, Shapiro \& Park, 2015). However, to the best of our knowledge, variability in the use of affect in the communication of the scientific content itself has not been investigated as a driver of public engagement.

The goals of this investigation are twofold. First, we seek to characterize the use of affect in TED talks, to understand the extent to which affect is utilized in TED talks relative to other science communication media. Second, we seek to elucidate whether the use of affect is related to engagement with science communication in TED talks. To this end, we generated a large database of TED talk transcripts and associated engagement data from YouTube. In the next section, we describe this database and our methodological approach to analyzing affect and engagement. Thereafter, we turn to the analysis of our main questions.

\section{A Database of TED Talk Transcripts and Engagement on YouTube}

We downloaded all available transcripts and corresponding information (e.g., title, presenter, tags) of TED videos from www.ted.com $(N=6,304)$. In processing the transcripts, we eliminated interview sections that frequently followed the presentations. This also led us to remove 465 transcripts that consisted exclusively of interviews, leaving 5,839 transcripts for further analysis. We obtained associated engagement data using the YouTube API. We retrieved all available engagement data, which included the number of views, likes, dislikes, and comments, for all 3,545 videos published by the TED YouTube channel. We then matched the transcripts and engagement data using the talk titles. Entries were matched using two strategies. First, we identified 2,475 exact title matches. Then, we looked for matches in the remaining 1,070 using approximate string matching and manual checking. This was necessary because many talks are published on YouTube using a different title than is used on the TED website. An additional 487 matches could thus be identified, amounting to a total of 2,962 complete entries. These were published between early 2007 and the end of 2020. The data were obtained on December 29th, 2020.

\section{Identifying Science in TED Talk Transcripts}

Although TED talks are widely considered a form of science communication (e.g., Sugimoto \& Thelwall, 2013), not all talks are science talks in the strict sense of the word. This is in part due to the large diversity of TED speakers. Apart from academics, many speakers are celebrities, journalists, athletes, and activists, to name a few categories. In a study conducted by MacKrill et al. (2021) that examined TED talks from 2006 to 2017, the authors found that only $27.4 \%$ of all talks were given by academics (i.e., people with a higher education degree and affiliated with a university). Past work on TED talks has addressed the diversity of speakers and content by using the topic tags that the TED organization assigned to each talk to characterize its content. For instance, Sugimoto and Thelwall (2013) used four of the 10 most frequent TEDassigned talk tags — science, technology, arts, and design — to distinguish between the two topics Art \& Design and Science \& Technology.

Using a similar approach, we infered topics from talk tags bottom-up using semangtic network analysis (Kenett et al., 2020. Siew et al., 2019). Specifically, we utilized the cooccurrences of talk tags (e.g. physics or medicine) to identify talk topics on the basis of homogeneous groups of tags (for a similar approach, see Wulff \& Mata, 2022). There were a total of 447 tags of which, on average, 8.2 different tags were assigned to each talk. Our approach to identifying science in TED talks consisted of four steps. In a first step,

\footnotetext{
${ }^{1}$ Retrieved from socialblade.com on August 7, 2021.
} 
we determined the relatedness of each pair of tags using the Jaccard similarity The Jaccard similarity measures the relatedness between tags by relating the number of TED talks for which the two tags co-occurred to the number of TED talks for which either of the tags occurred:

$$
J(A, B)=1-\frac{|A \bigcap B|}{|A \bigcup B|}
$$

In a second step, we used the relatedness of tag pairs to construct a weighted network of tags and apply the Louvain modularity detection algorithm as implemented in the igraph R package (Csardi \& Nepusz, 2006) to identify homogenous groups of tags within the network (Blondel et al., 2008. Haslbeck \& Wulff, 2020). Note that the Louvain algorithm has been found to compare favorably to other modularity and clustering algorithms (e.g., Emmons et al., 2016 Miasnikof et al., 2020; Pradana et al., 2020, Williams et al., 2019). The algorithm produced seven groups (hereinafter referred to as topics), which we labeled Mind, Entertainment, Tech, Health, Cosmos, Environment, and Society.

In a third step, we substituted tags with their topic assignments and used the maximum positive point-wise mutual information, a common metric to assess the strength of semantic relationships (Bullinaria \& Levy, 2007), between talks and topics to assign each talk to one of our seven topics.

To assess the quality of the mapping between talks and topics, we conducted a text-analysis of talk titles. Specifically, again using point-wise mutual information, we determined the most relevant words in TED talk titles for each of the topics (see Figure 1. We found the titles of talks assigned to Mind to contain words such as "depressed", "compassion", or "decisions", those assigned to Entertainment contain words such as "comedy", "poetry", or "violin", those assigned to Tech to contain words such as "hacked", "computers, or "net", those assigned to Health to contain words such as "synthetic", "diseases", or "antibiotics", those assigned to Cosmos to contain words such as "planets", "galaxies", or "mars", those assigned to Environment to contain words such as "ocean", "trees", or "sustainable", and, finally, those assigned to Society to contain words such as "gun", "immigration", or "corruption". We further utilized a pre-trained sentence embedding, the Universal Sentence Encoder (Cer et al., 2018), to compare the semantic similarity of talk titles from the same topics to those of different topics. We found the within-topic similarity to exceed the between-topic similarity for every topic, with the difference in terms of Cohen's $d$ ranging from .18 (Entertainment) to .69 (Cosmos) Together, these results speak for an accurate mapping of talks to semantically distinct topics.

Fourth and finally, to address the question which set of TED talks most concerns science communication, we computed a science index for each of the seven topics. This index reflected the percent of talks in each of the seven topics that either were assigned the tag Science or contained the words "science", "experiment", or "study" within the transcript. Using this index, we found that the topic Health (79\%) was most linked to science, followed by Cosmos (78\%), Mind (63\%), Mind (69\%), Tech 58\%, Society (43\%), and Entertainment $(37 \%)$.

\section{Sentiment Analysis}

To identify affect expressed in TED talk transcripts, we relied on a dictionary-based approach (Denecke, 2008), which is a very common approach in sentiment analysis (Feldman, 2013. Medhat et al., 2014). It involves mapping, wherever possible, the words in a text - the talk transcripts - to their corresponding sentiment value in the dictionary and calculating summaries of these values. In contrast to previous approaches, which often made use of the proprietary Linguistic Inquiry and Word Count (LIWC) database (e.g., Berger \& Milkman, 2012; Brady et al., 2017; Hwong et al., 2017; Milkman \& Berger, 2014), we relied on the openly available SentiWordNet sentiment dictionary (Baccianella et al., 2010). It contains more than 20,000 words with affect values ranging from -1 (most negative) to 1 (most positive). Similar to other sentiment dictionaries, SentiWordNet contains more negative $(55 \%)$ than positive $(45 \%)$ words, resulting in a negative average value of $-0.06(S D=.34)$. Using SentiWordNet, we calculated two sentiment summaries of the sentiment values $s$ for each transcript. First, to capture whether the speaker used predominantly positive or negative words, we calculated an affective valence score

$$
\text { valence }=\frac{1}{n} \sum_{i}^{n} s
$$

where $n$ is the total number of sentiment values available in a transcript. Second, to capture the speaker's tendency to rely on affect-laden words, irrespective of whether they have positive or negative valence, we calculated an affective density score

$$
\text { density }=\frac{1}{n} \sum_{i}^{n} I(s \neq 0)
$$

where $I()$ is an indicator function assigning a value of 1 when $s \neq 0$ and a value of 0 when $s=0$. To our knowledge, the distinction of sentiment valence and density represents a novel contribution of this investigation, although related notions of sentiment density have been discussed in the literature (see Dong et al., 2013; Liu et al., 2018; Varshney \& Wagh, 2017)

\section{Dimensions of Engagement}

Past work seeking to quantify engagement on social media has mostly focused on combined engagement scores, calcu- 

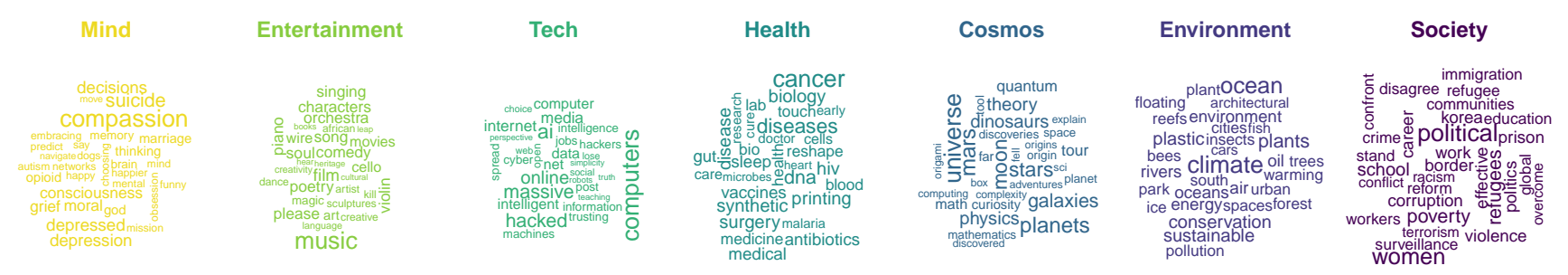

Figure 1

Most relevant words in TED talk titles per topic. Size of the words reflects the positive point-wise mutual information between the words and the topics derived from the network of tag co-occurrences. The 30 most relevant words per topic are displayed.

lated as a weighted sum of all available aspects of engagement (e.g., Hwong et al., 2017; Kim \& Yang, 2017; Kujur \& Singh, 2018, Vadivu \& Neelamalar, 2015). Such approaches are sensible in light of typically strong correlations between engagement variables and can simplify matters in situations where the main goal is to generate a single metric capturing overall engagement. Recent investigations have, however, highlighted the value of distinguishing between different types of engagement. For instance, Srinivasan et al. (2013) found that image posts tend to garner more likes than comments, whereas the opposite was true for text posts. We therefore decided not to rely on a single engagement measure, but instead to use a data-driven approach to summarize the engagement variables available. Specifically, we used principal component analysis to extract independent, synthetic engagement variables from four variables of engagement available through the YouTube API: views, the number of times a video was clicked on; likes, the number of times viewers clicked the like button; dislikes, the number of times viewers clicked the dislike button; and comments, the number of times viewers left a comment. These variables were highly correlated $(.70<r<.92)$, due to the fact that likes, dislikes, and comments are secondary to a video being viewed. We found that two engagement components were able to account for $95.4 \%$ of the total variance (see Figure 2). The first engagement component, which we labeled popularity, captured positive reactions in the form of views and likes, whereas the second engagement component, labeled polarity, captured negative or contrarian reactions in the form of dislikes and comments.

\section{Exploring the Use of Affect in TED Talks}

To gain insight into how affect is used in TED talks, before analyzing how this is linked to engagement, we took two approaches. First, we compared the values of affective valence and density in TED talk transcripts to those in other media based on text or video. Specifically, we compared TED talks to a random subset of 1,000 scientific articles on the preprint server arXi设 which primarily report research on STEM topics, a random subset of 1,000 scien- tific articles from the journal Psychological Science 3 , which report results on all topics in psychology, including research on emotion and affect, and random samples of text sources of other media, including Wikipedia articles, news articles, and subtitles of TV shows, soap operas, and movies 4 . This analysis revealed that the use of affect in TED talks is distinct from all reference media (see Figure $3 \mathrm{~A}$ ). They show considerably higher affective valence and, in particular, higher density than all text-based media-academic articles from arXiv and Psychological Science articles, books, Wikipedia articles, and news articles-but also lower affect and density than all video-based media-movies, TV shows, and soap operas. The analysis also revealed that the use of affect in TED talks is, on average, more similar to that in other videobased media than that in text-based media, especially considering traditional expert-to-expert science communication in the form of academic articles. Nevertheless, there was also considerable variance in the use of affect in TED talks, spanning the full gamut between text and video-based media.

Second, we analyzed the valence and density of TED talks as a function of the publishing year and topic, in order to assess whether the use of affect in TED talks has been stable

\footnotetext{
${ }^{2}$ Downloaded from www.kaggle.com/Cornell-University/arxiv

${ }^{3}$ Downloaded from the journal's homepage.

${ }^{4}$ Obtained from https://www.english-corpora.org/
}

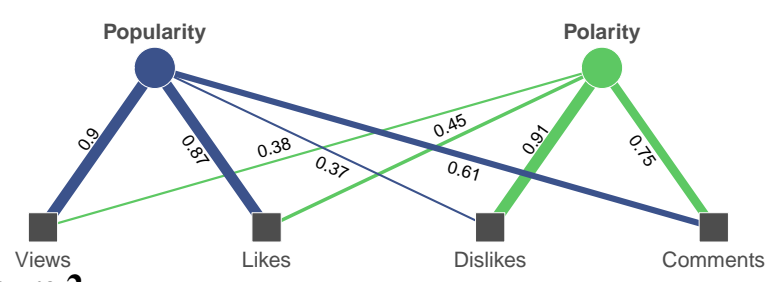

Figure 2

Composition of engagement components. The figure shows the loadings of the four engagement variables on two principal components that constitute two forms of engagement: popularity and polarity. The presented solution accounts for 95.4\% of the variance of the four manifest engagement variables. 
over time and is independent of the topic of TED talks (see 3B-E). This analysis revealed that the valence in TED talks has decreased since 2007, whereas density seems to have increased, at least in recent years. Furthermore, the analysis showed that there were noticeable differences in the use of affect between topics. Affective valence was most positive in Entertainment, followed by Tech, Cosmos, Mind, Health, Society, and Environment, whereas affective density was highest in Mind, followed by Health, Tech, Environment, Entertainment, Society, and Cosmos. Finally, we also analyzed the link between publishing year and topic. This revealed that talks on Society, Health, and Environment have become more frequent at the expense of, in particular, talks on Entertainment, which may account for the temporal trends in the use of affect across time.

In sum, the language in TED talks contains elevated levels of affect valence and density that are more similar to video-based than text-based media, including those reflecting expert-to-expert science communication in the form of academic articles. Furthermore, there was considerable variance in the use of affect in TED talks, of which some is accounted for by differences in publishing years and topics. In the next section, we will explore whether the variance in the use of affect corresponds with engagement with year and topic controlled for.

\section{The Link between Affect and Engagement with TED Talks}

To evaluate the role of affect for engagement with TED talks, we ran separate regression analyses for our two engagement components. As predictors, we included valence and density as well as two sets of covariates: First, to control for the differences in the use of affect presented in the previous section, we included the talk topic and publishing date on YouTube. The inclusion of publishing date further allowed us to control for differences in engagement, in particular, concerning the number of views, which varies as a function of a video's age at data collection.. Second, to also control for other factors that might drive engagement besides affect, we included the duration of the video and the Flesch Reading Ease score, which captures the accessibility of the language used in the talk (Flesch, 1948). The analysis of popularity revealed that more positive valence and higher density was associated with higher popularity. The effect of density $(d=.21)$ was twice as large as the effect of valence $(d=.12)$; however, both effects are small in magnitude. High popularity was also associated with long duration $(d=.32)$, high readability $(d=.20)$, and the topic Mind, in contrast to all other topics and especially the topics Environment and Society, which were associated with low popularity. The analysis of polarity, by contrast, revealed a negative association with valence $(d=-.83)$, but not density $(d=.02)$. The effect of valence implies that more negative valences were associ- ated with high polarity; however, this effect was again small in magnitude. High polarity was also associated with longer duration $(d=.20)$ and with the topic Society in contrast to, in particular, Health, Cosmos and Environment, which were negative in polarity.

To address whether the effects engagement generalize across topics, we reran the regression analyses separately for each of the seven topics and the twelve most relevant tags within each topic. Specifically, we compared the effects of affect on engagement for talks with a given topic or tag against talks without the topic or tag, using models that included all other predictors presented above except (see Table 11. In other words, we evaluated by how much and in which direction the content of talks moderates the effect of affect on engagement. Figure 5 illustrates this moderation in terms of Cohen's $d$ for the two engagement variables, popularity and polarity, and the two content levels, topics and tags. The results reveal considerable moderation for some but not all content. Beginning with popularity (Panels A and C), talks from the topic Environment, especially those with tags "Green" or "Sustainability", show a noticeable reduction in the effect of density on popularity. The strength of the reduction implies that density in talks from the topic Environment was no longer related to popularity $(d=-.02)$. The opposite, an increase in the link between density and popularity, was the case for talks from the topic Mind, especially for talks with tags "Decision-Making" or "Mental Health". Furthermore, talks from the topic Society showed an elevated effect of valence, in particular talks with tags "Immigration" or "Refugees", with valence in these talks being considerably more strongly related to popularity than in talks from other topics. We observed the opposite for talks from the topic Health, in particular talks with tags "Medicine" or "DNA", with the result that valence was related mildly negatively to popularity $(d=-.07)$ within Health-related talks. Compared to these four topics, Cosmos, Tech, and Entertainment showed smaller levels of moderation for popularity.

Turning to polarity (Panels B and D), talks from the topic Tech, especially those with tags "AI" and "Machine Learning", and talks from Environment, especially with tags "Green" and "Sustainability", showed increased effects of density as compared to talks from other topics, resulting in strong positive associations with polarity within these topics (Tech: $d=.49$, Environment: $d=.27$ ), whereas density in talks from Society, especially with tags "Refugees" and "Criminal Justice", showed a reduction in the effect of density on polarity, resulting in a small negative effect within this topic $(d=-.17)$. Furthermore, talks from the topic Entertainment showed an increase in the effect of valence on polarity, with more positive valence being associated with a small increase in polarity $(d=.14)$. In comparison, talks from the topics Mind, Cosmos, and Health showed smaller moderation effects for polarity. 


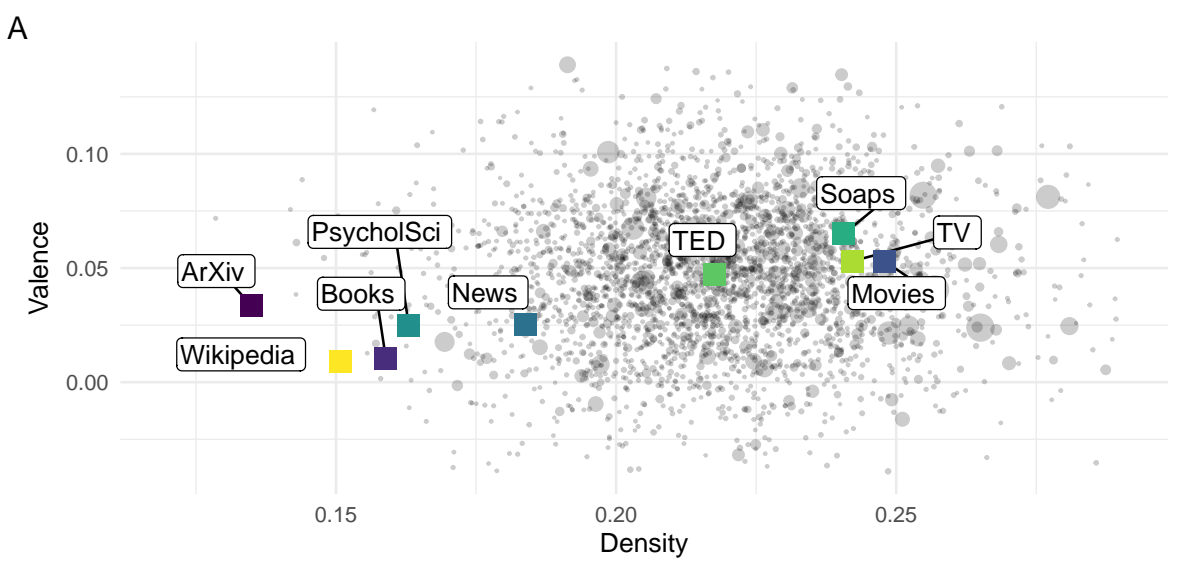

B

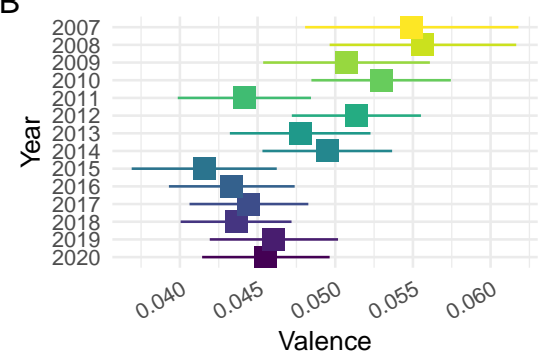

D

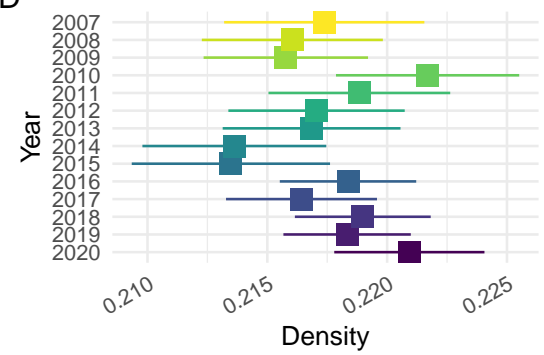

C

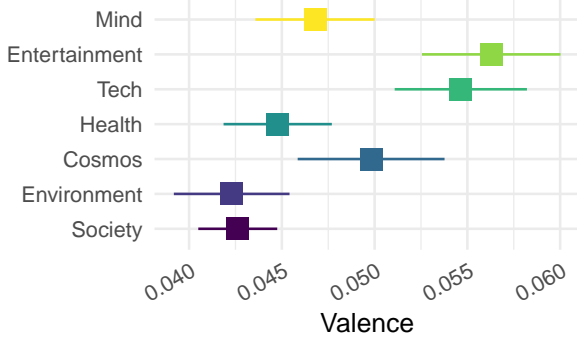

$\mathrm{E}$

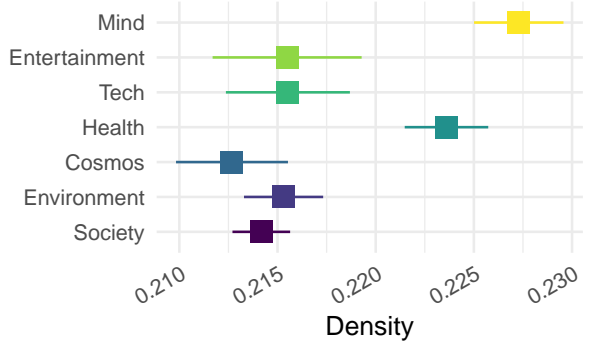

\section{Figure 3}

Affect valence and density of TED talks. Panel A shows as circles in the background the valence and density of all 2,962 TED talks, with their size scaled according to number of views. The squares in the foreground show the average valence and density of the TED talks and the reference texts (see section A Characterization of Affect in TED Talks). Panels B-E show the average valence and density separately for each publishing year and topic. For details on the topic extraction see the section Identifying Science in TED talks.

Finally, we also analyzed moderation with respect to the science index described above. We observed a small moderation effect for popularity, with talks of scientific content exhibiting a slightly reduced association of valence and popularity and a slightly increased association of density and popularity. For polarity, no moderation was observed. Consequently, the effect of affect on engagement was largely unchanged for talks with a positive science index. Valence remained positively related to popularity $(d=.06)$ and negatively to polarity $(d=-.68)$, whereas density was more strongly related to popularity $(d=.29)$, while remaining to be unrelated to polarity $(d=.02)$.
In sum, affective valence and density were significantly linked to engagement with TED talks on YouTube, with increased valence and density being associated with increased popularity, and increased valence but not density being associated with negative polarity. These links were moderated by topics, with some topics seeing significantly pronounced or reversed relationships, suggesting that the link between affect and engagement depends in parts on the content of talks. However, we did not observe meaningful moderation as a function of the science index, suggesting that the moderation by content is independent on whether the content focuses on science or not. 
A

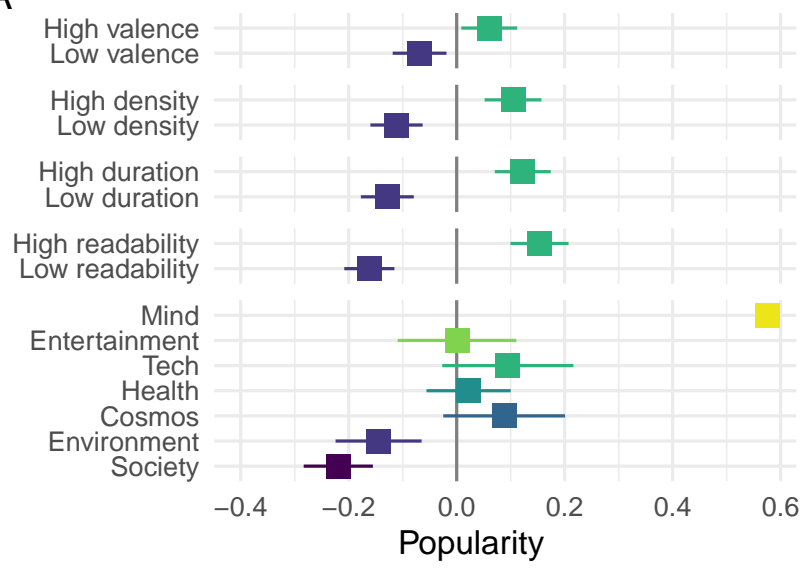

B

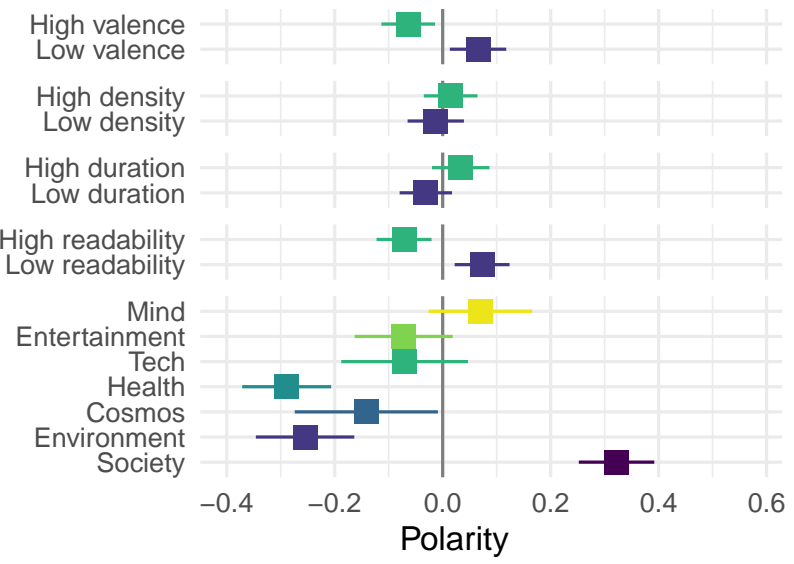

\section{Figure 4}

Engagement as a function of the talks' valence, density, duration, readability, and topic. High and low value groups were created by median split.

Table 1

Predicting the popularity and polarity of TED talks

\begin{tabular}{llllllll}
\hline & \multicolumn{3}{c}{ Popularity } & \multicolumn{3}{c}{ Polarity } \\
& $d$ & $F$ & $p$ & $d$ & $F$ & $p$ \\
\hline Affect & & & & & & \\
Valence & .12 & 9.68 & .002 & -.08 & 5.05 & .024 \\
Density & .21 & 32.21 & $<.001$ & .02 & 0.29 & .589 \\
Control & & & & & & \\
Duration & .32 & 72.08 & $<.001$ & .20 & 28.36 & $<.001$ \\
Readability .20 & 30.43 & $<.001$ & -.06 & 2.85 & .914 \\
Topic & .42 & 21.74 & $<.001$ & .43 & 22.58 & $<.001$ \\
Date & -.05 & 1.76 & .184 & .51 & 188.07 & $<.001$ \\
\hline
\end{tabular}

\section{Discussion}

Scientists increasingly participate in the communication of science to the public. One example of this are TED talks, where researchers give short presentations directed at a broad audience that are recorded and then made accessible and shared via social media. Science communication can thereby reach audiences far beyond the scientific community, provided the communication is done effectively. Here, we investigated the role of affect as one potential moderator of effective science communication in the context of social media. To do this, we analyzed how affect expressed in the transcripts of TED talks corresponds with engagement on YouTube. First, we observed that the use of affect in TED talks in terms of valence and density is more similar to affect-laden visual media, such as movies or soap operas, than to traditional text-based media, such as books, news articles, and academic articles. Second, we observed that the two measures of affect were significantly related to two com- ponents of engagement: popularity and polarity. Higher affective valence was associated with higher popularity, reflecting more views and likes, and lower polarity, reflecting fewer dislikes and comments. Higher affective density, on the other hand, was related to higher popularity for almost all topics. Third we observed substantial moderation of these effects by the topic of the talk, but not whether the talk contained scientific or non-scientific content.

Our results demonstrate that affect as a surface-level characteristic of science communication on social media can impact how the public engages with its content. At least two explanations for this link come to mind. First, higher levels of affect may heighten or lower the mood of the audience or alter its level of arousal, and thereby impact engagement. Second, higher levels of affect may signal more opinionated and assertive positions that increase the likelihood for engagement, both supportive or critical. It seems plausible that both of the accounts are, at least, partially true. On the one hand, associations of mood or arousal with engagement in social media are well-document (e.g., De Choudhury et al., 2012, Kujur \& Singh, 2018; Osseweijer, 2006; Schreiner et al., 2021). On the other hand, moderation of the association between affect and engagement was particularly pronounced for controversial or disruptive topics, such as "Refugees", "AI", "Sustainability", or "Health care", where the audience may variably favor a opinionated or a more measured approach (Hall et al., 2018, Hertwig \& Wulff, 2021).

Our results have immediate practical implications for science communication on YouTube and similar social media outlets. They suggest that communicators can, possibly, utilize the two components of affect to increase the public's (social media) engagement with their content. Specifically, if science communicators incorporate more affect-laden words 
A

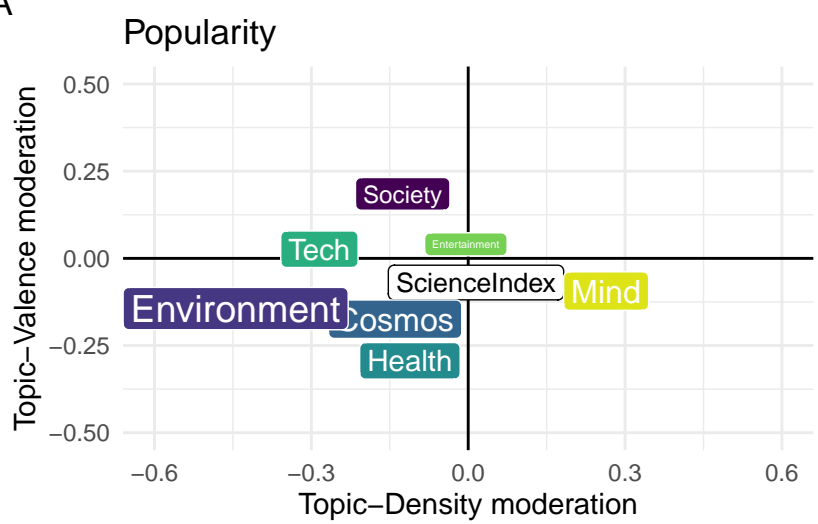

C

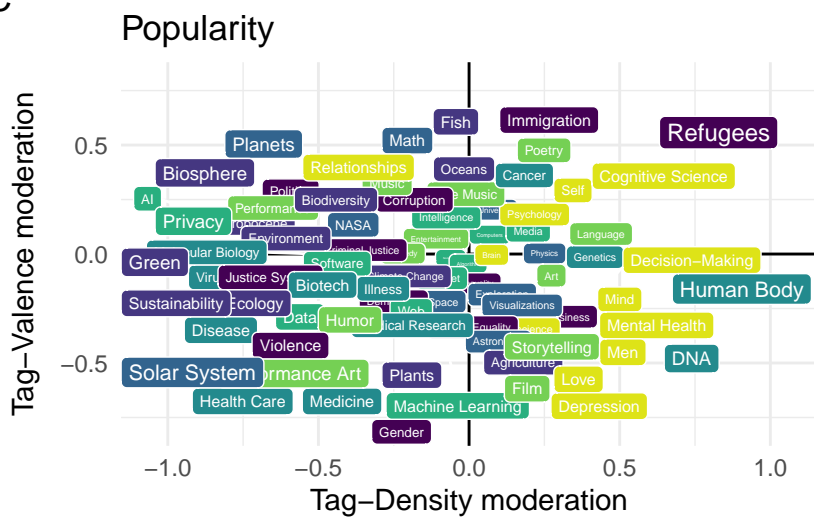

B

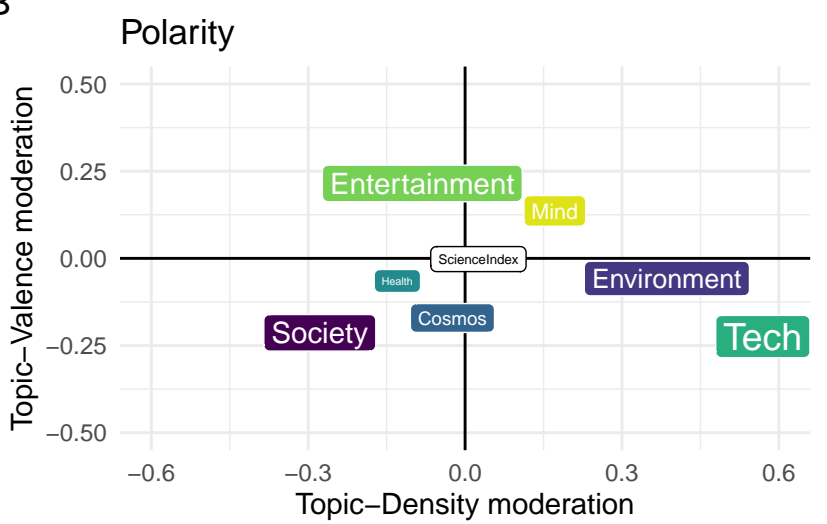

D
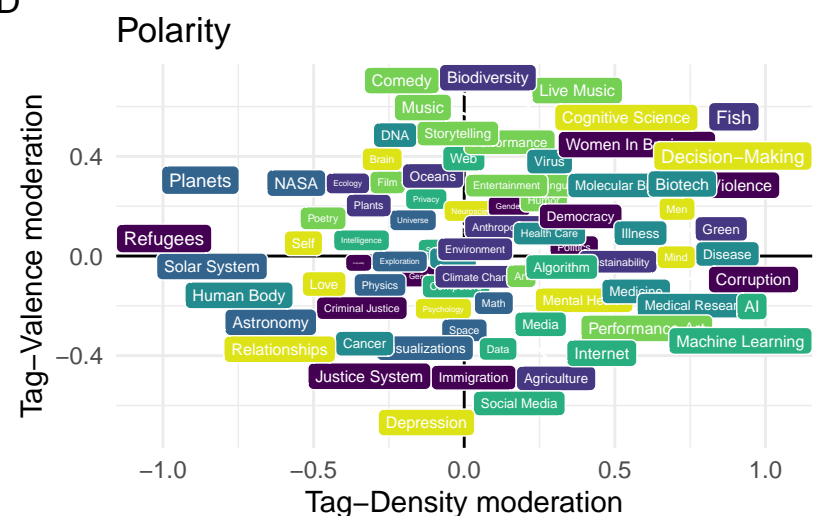

Figure 5

Moderation of the effect of affect on engagement by talk topic and talk tag. The figure shows the difference in effect size d between the effects of affect on engagement for talks with given topic label or tag versus those without the topic or label. Positive values indicate that talks with the topic label or tag show a more positive effect than talks with the label or tag. Panels $A$ and $C$ show the results for models predicting popularity, whereas panels $B$ and $D$ show the corresponding results for polarity. Color reflects topic and size the magnitude of moderation across both, density and valence.

overall (i.e., higher density) and more positive rather than negative affect words in particular (i.e., higher valence), their content may receive more views and likes (i.e., higher popularity) as well as fewer dislikes and comments (i.e., lower polarity as a result of higher valence). However, although increasing the valence and density of one's content may lead to an increase of that content's popularity on average, this effect may not generalize to all types of content. Making a talk more positive (i.e., higher valence) may in some instances backfire, for instance when the talk already has high valence or when it does not meet the expectations of the audience. Furthermore, although higher density of affect in talks is linked to higher popularity in almost all cases, simply increasing the density of affect in one's talk without considering one's overall use of language (e.g., use of jargon, visual imagery, story arc) may not yield the desired effects. Therefore, it is essential that science communicators become aware that the way in which they communicate science does indeed influence its reception and dissemination beyond the scientific community. In other words, to disseminate scientific findings to a broader audience, scientists may need — and are, perhaps, already expected — to become "fluent" in the many languages of science communication beyond traditional publications (e.g., blog posts or video essays; see Ho et al.,2021. for a discussion of science communication in other formats).

Our study has several limitations that deserve discussion. First, our study relies on a purely correlative design. As a consequence, we can only speculate as to the causal mechanisms underlying our results and must refer to future experimental work to settle the above-mentioned hypotheses. Second, TED talks are a particular form of public science communication (MacKrill et al., 2021; Sugimoto \& Thelwall, 2013, Verjovsky \& Jurberg, 2020) that likely differs from other kinds, especially those based purely on text, which we found to rely much less on affective language. It is therefore unclear to what extent our findings translate to, for instance, 
academic posts on social media (Rohrer et al., 2021) or traditional press releases. Third, and relatedly, TED talks are unusual in that they are not exclusively used by academics to communicate scientific content, but also used by other professionals for presentations that may or may not relate to science. The presence of other content may imply that the scientific content is evaluated differently than it would be in a medium that is geared exclusively towards science communication. However, the presence of alternative, rivaling content is probably not unique to TED talks, but likely a characteristic of most media used for public science communication. Fourth, the engagement variables available to us did not include shares, which are an arguably stronger and more participatory form of engagement than the engagement variables in our analysis and are important for the dissemination of content on social media (Shao, 2009). It is probable that shares would fall into our popularity component, given that they have been linked to higher ratings of scientific content's interestingness and usefulness-suggesting that shares often express support - as well as higher ratings of emotionality (a subjective measure similar to density) and positivity (an objective measure similar to valence; see Milkman \& Berger, 2014). Accordingly, we would expect that talks with higher valence and density would have received more shares.

\section{Conclusion}

Our analysis of TED talks establishes affect as an important driver of lay audience engagement with science communication. A talk's affective valence and density are distinctly related to audience engagement in terms of both popularity and polarity, with the talk's topic taking a moderating role. We have outlined several directions that may help improve understanding of the exact mechanisms underlying the influence of affect on engagement. Nevertheless, our results highlight that science communicators should be aware that surface-level aspects such as affect could co-determine engagement and reach among lay audiences on social media.

\section{Declarations}

\section{Ethical approval and informed consent}

This article does not contain any studies with human participants performed by any of the author.

\section{Competing interest}

The authors declare no competing interests.

\section{Data availability}

The datasets generated during and analyzed in this study are available at this repository (link added after review).

\section{References}

Aldous, K. K., An, J., \& Jansen, B. J. (2019). View, Like, Comment, Post: Analyzing User Engagement by Topic at 4 Levels across 5 Social Media Platforms for 53 News Organizations. Proceedings of the International AAAI Conference on Web and Social Media, 13, 47-57. Retrieved January 21, 2021, from https://ojs.aaai.org/index.php/ICWSM/article/view/ 3208

Allgaier, J. (2020). Science and medicine on youtube. Second international handbook of Internet research, 7-27.

Amarasekara, I., \& Grant, W. J. (2019). Exploring the youtube science communication gender gap: A sentiment analysis. Public Understanding of Science, 28(1), 68-84.

Baccianella, S., Esuli, A., \& Sebastiani, F. (2010). Sentiwordnet 3.0: An enhanced lexical resource for sentiment analysis and opinion mining. Lrec, 10(2010), 2200-2204

Berger, J., \& Milkman, K. L. (2012). What Makes Online Content Viral? Journal of Marketing Research, 49(2), 192-205.

Blondel, V. D., Guillaume, J.-L., Lambiotte, R., \& Lefebvre, E. (2008). Fast unfolding of communities in large networks. Journal of statistical mechanics: theory and experiment, 2008(10), P10008.

Boczkowski, P., Mitchelstein, E., \& Matassi, M. (2017). Incidental news: How young people consume news on social media. Proceedings of the 50th Hawaii international conference on system sciences.

Boy, B., Bucher, H.-J., \& Christ, K. (2020). Audiovisual Science Communication on TV and YouTube. How Recipients Understand and Evaluate Science Videos. Frontiers in Communication, 5, 608620. https://doi.org/10.3389/fcomm.2020.608620

Brady, W. J., Wills, J. A., Jost, J. T., Tucker, J. A., \& Van Bavel, J. J. (2017). Emotion shapes the diffusion of moralized content in social networks. Proceedings of the National Academy of Sciences, 114(28), 7313-7318. https : / / doi . org / 10 . 1073 / pnas . 1618923114

Brossard, D. (2013). New media landscapes and the science information consumer. Proceedings of the National Academy of Sciences, 110, 14096-14101. https:// doi.org/10.1073/pnas.1212744110

Bullinaria, J. A., \& Levy, J. P. (2007). Extracting semantic representations from word co-occurrence statistics: A computational study. Behavior research methods, 39(3), 510-526.

Cer, D., Yang, Y., Kong, S.-y., Hua, N., Limtiaco, N., John, R. S., Constant, N., Guajardo-Cespedes, M., Yuan, S., Tar, C., et al. (2018). Universal sentence encoder. arXiv preprint arXiv:1803.11175. 
Collins, K., Shiffman, D., \& Rock, J. (2016). How are scientists using social media in the workplace? PloS one, 11(10), e0162680.

Covington, P., Adams, J., \& Sargin, E. (2016). Deep neural networks for youtube recommendations. Proceedings of the 10th ACM conference on recommender systems, 191-198.

Csardi, G., \& Nepusz, T. (2006). The igraph software package for complex network research. InterJournal, Complex Systems, 1695. https://igraph.org

Davidson, J., Liebald, B., Liu, J., Nandy, P., Van Vleet, T., Gargi, U., Gupta, S., He, Y., Lambert, M., Livingston, B., et al. (2010). The youtube video recommendation system. Proceedings of the fourth ACM conference on Recommender systems, 293-296.

Davies, S. R. (2019). Science communication as emotion work: Negotiating curiosity and wonder at a science festival. Science as Culture, 28(4), 538-561.

Davies, S. R., Halpern, M., Horst, M., Kirby, D. S., \& Lewenstein, B. (2019). Science stories as culture: Experience, identity, narrative and emotion in public communication of science.

De Choudhury, M., Counts, S., \& Gamon, M. (2012). Not all moods are created equal! exploring human emotional states in social media. Proceedings of the International AAAI Conference on Web and Social Media, 6(1), 66-73.

Denecke, K. (2008). Using sentiwordnet for multilingual sentiment analysis. 2008 IEEE 24th international conference on data engineering workshop, 507512.

Dong, R., Schaal, M., O’Mahony, M. P., McCarthy, K., \& Smyth, B. (2013). Mining features and sentiment from review experiences. International Conference on Case-Based Reasoning, 59-73.

Emmons, S., Kobourov, S., Gallant, M., \& Börner, K. (2016). Analysis of network clustering algorithms and cluster quality metrics at scale. PloS one, 11(7), e0159161.

Feldman, R. (2013). Techniques and applications for sentiment analysis. Communications of the ACM, 56(4), 82-89.

Flesch, R. (1948). A new readability yardstick. Journal of applied psychology, 32(3), 221-233.

Fronzetti Colladon, A., D’Angelo, C. A., \& Gloor, P. A. (2020). Predicting the future success of scientific publications through social network and semantic analysis. Scientometrics, 124(1), 357-377.

Gheorghiu, A. I., Callan, M. J., \& Skylark, W. J. (2020). A Thin Slice of Science Communication: Are People's Evaluations of TED Talks Predicted by Superficial Impressions of the Speakers? Social Psycho- logical and Personality Science, 11(1), 117-125. https://doi.org/10.1177/1948550618810896

Hall, M. G., Sheeran, P., Noar, S. M., Boynton, M. H., Ribisl, K. M., Parada Jr, H., Johnson, T. O., \& Brewer, N. T. (2018). Negative affect, message reactance and perceived risk: How do pictorial cigarette pack warnings change quit intentions? Tobacco control, 27(e2), e136-e142.

Haslbeck, J. M., \& Wulff, D. U. (2020). Estimating the number of clusters via a corrected clustering instability. Computational Statistics, 35, 1879-1894.

Hertwig, R., \& Wulff, D. U. (2021). A descriptionexperience framework of the psychology of risk. Perspectives on psychological science.

Ho, M.-T., Ho, M.-T., \& Vuong, Q.-H. (2021). Total SciComm: A Strategy for Communicating Open Science. Publications, 9(3), 31. https://doi .org/10. 3390/publications9030031

Hoiles, W., Aprem, A., \& Krishnamurthy, V. (2017). Engagement and popularity dynamics of youtube videos and sensitivity to meta-data. IEEE Transactions on Knowledge and Data Engineering, 29(7), 14261437.

Hwong, Y.-L., Oliver, C., Van Kranendonk, M., Sammut, C., $\&$ Seroussi, Y. (2017). What makes you tick? The psychology of social media engagement in space science communication. Computers in Human Behavior, 68, 480-492. https://doi.org/10.1016/j.chb. 2016.11 .068

Kaul, L., Schrögel, P., \& Humm, C. (2020). Environmental science communication for a young audience: A case study on the\# earthovershootday campaign on youtube. Frontiers in Communication, 5, 108.

Kenett, Y. N., Beckage, N. M., Siew, C. S., \& Wulff, D. U. (2020). Cognitive network science: A new frontier.

Kim, C., \& Yang, S.-U. (2017). Like, comment, and share on facebook: How each behavior differs from the other. Public Relations Review, 43(2), 441-449. https:// doi.org/10.1016/j.pubrev.2017.02.006

Kohler, S., \& Dietrich, T. C. (2021). Potentials and limitations of educational videos on youtube for science communication. Front. Commun. 6: 581302. doi: 10.3389/fcomm.

Kujur, F., \& Singh, S. (2018). Emotions as predictor for consumer engagement in YouTube advertisement. Journal of Advances in Management Research, 15(2), 184-197. https://doi.org/10.1108/JAMR05-2017-0065

formula to calculate engagement rate BUT: does not use "views" like we do

Liu, Z., Zhang, W., Cheng, H. N., Sun, J., \& Liu, S. (2018). Investigating relationship between discourse behavioral patterns and academic achievements of stu- 
dents in spoc discussion forum. International Journal of Distance Education Technologies (IJDET), 16(2), 37-50.

MacKrill, K., Silvester, C., Pennebaker, J. W., \& Petrie, K. J. (2021). What makes an idea worth spreading? language markers of popularity in $<$ span style="fontvariant:small-caps;" $>$ TED $</$ span $>$ talks by academics and other speakers. Journal of the Association for Information Science and Technology, 72(8), 1028-1038. https://doi.org/10.1002/asi.24471

Medhat, W., Hassan, A., \& Korashy, H. (2014). Sentiment analysis algorithms and applications: A survey. Ain Shams engineering journal, 5(4), 1093-1113.

Miasnikof, P., Shestopaloff, A. Y., Bonner, A. J., Lawryshyn, Y., \& Pardalos, P. M. (2020). A density-based statistical analysis of graph clustering algorithm performance. Journal of Complex Networks, 8(3), cnaa012.

Milkman, K. L., \& Berger, J. (2014). The science of sharing and the sharing of science. Proceedings of the National Academy of Sciences, 11(Supplement 4), 13642-13649. https : // doi . org / 10 . 1073/pnas . 1317511111

Osseweijer, P. (2006). A new model for science communication that takes ethical considerations into account: The three-e model: Entertainment, emotion and education. Science and Engineering Ethics, 12(4), 591.

Pradana, C., Kusumawardani, S., \& Permanasari, A. (2020). Comparison clustering performance based on moodle log mining. IOP Conference Series: Materials Science and Engineering, 722(1), 012012.

Rakedzon, T., Segev, E., Chapnik, N., Yosef, R., \& BaramTsabari, A. (2017). Automatic jargon identifier for scientists engaging with the public and science communication educators. PloS one, 12(8), e0181742.

Rohrer, J. M., Tierney, W., Uhlmann, E. L., DeBruine, L. M., Heyman, T., Jones, B., Schmukle, S. C., Silberzahn, R., Willén, R. M., Carlsson, R., et al. (2021). Putting the self in self-correction: Findings from the lossof-confidence project. Perspectives on Psychological Science.

Schreiner, M., Fischer, T., \& Riedl, R. (2021). Impact of content characteristics and emotion on behavioral engagement in social media: Literature review and research agenda. Electronic Commerce Research, 21(2), 329-345.

Shao, G. (2009). Understanding the appeal of user-generated media: A uses and gratification perspective [Publisher: Emerald Group Publishing Limited]. Inter- net Research, 19(1), 7-25. https://doi.org/10.1108/ 10662240910927795

Shapiro, M. A., \& Park, H. W. (2015). More than entertainment: Youtube and public responses to the science of global warming and climate change. Social Science Information, 54(1), 115-145.

Sharon, A. J., \& Baram-Tsabari, A. (2014). Measuring mumbo jumbo: A preliminary quantification of the use of jargon in science communication. Public Understanding of Science, 23(5), 528-546. https://doi. org/10.1177/0963662512469916

Siew, C. S., Wulff, D. U., Beckage, N. M., \& Kenett, Y. N. (2019). Cognitive network science: A review of research on cognition through the lens of network representations, processes, and dynamics. Complexity, 2019.

Srinivasan, B. V., Natarajan, A., Sinha, R., Gupta, V., Revankar, S., \& Ravindran, B. (2013). Will your facebook post be engaging? Proceedings of the 1st Workshop on User Engagement Optimization UEO '13, 25-28. https://doi.org/10.1145/2512875. 2512881

Sugimoto, C. R., \& Thelwall, M. (2013). Scholars on soap boxes: Science communication and dissemination in TED videos. Journal of the American Society for Information Science and Technology, 64(4), 663674. https://doi.org/10.1002/asi.22764

Vadivu, V. M., \& Neelamalar, M. (2015). Digital brand management - A study on the factors affecting customers' engagement in Facebook pages. 2015 International Conference on Smart Technologies and Management for Computing, Communication, Controls, Energy and Materials (ICSTM), 71-75. https: //doi.org/10.1109/ICSTM.2015.7225392

Varshney, V., \& Wagh, R. S. (2017). Weighted sentiment score formulation using sentence level sentiment density for opinion analysis. International Journal of Computational Intelligence Research, 13(2), 285-298.

Verjovsky, M., \& Jurberg, C. (2020). Spreading ideas: TED talks' role in cancer communication and public engagement. Journal of Cancer Education, 35(6), 1206-1218. https://doi.org/10.1007/s13187-01901583-6

Williams, N., Arnulfo, G., Wang, S. H., Nobili, L., Palva, S., \& Palva, J. M. (2019). Comparison of methods to identify modules in noisy or incomplete brain networks. Brain connectivity, 9(2), 128-143.

Wulff, D. U., \& Mata, R. (2022). On the semantic representation of risk. Science Advances. 